\title{
Repair and restore: make a difference
}

\author{
Chandrasekar Padmanabhan ${ }^{1}$ (D)
}

Received: 20 November 2019 / Accepted: 25 November 2019/Published online: 4 January 2020

(C) Indian Association of Cardiovascular-Thoracic Surgeons 2020

The field of valve repair has evolved from being intuitive and surgeon-centric, to one with objective and reproducible formats. Valve repair now stands in an era with long-term positive outcomes and freedom from re-operation. Though the science of valvular heart surgery has witnessed steady advancement over the years, it still presents a challenge to the surgeon.

With various schools of thought across this landscape, and each one claiming superiority, it becomes of paramount importance for the modern surgeon to have a clear understanding of current ideas, concepts, and techniques, and the data behind these advancements.

This issue attempts to present a comprehensive review on valve repair in the modern sense. With contributions from reputed surgeons and cardiologists with extensive experience in their respective fields, I hope the material provided here will help each surgeon have a better understanding of the science behind valve repair and its application in current clinical practice.

\section{The mitral valve}

Historically, the mitral valve has received maximum attention in terms of reconstruction. Modern reparative techniques were initiated in the landmark article "The French Correction", published in 1983 by Professor Alain Carpentier, which demonstrated the functional approach and caused a paradigm shift in practice - from simply replacing the valve to preserving it.

In India, though it still presents a problem, rheumatic heart disease is slowly declining in prevalence; with the aging

Chandrasekar Padmanabhan

chanpad@gmail.com

1 Department of cardiothoracic Surgery, G. Kuppuswamy Naidu Memorial Hospital, P.N. Palayam, Coimbatore 641037, India population demonstrating a more degenerative pathology compared to decades past. Also, as seen in other parts of the world, occurrence of heart failure is growing, aided in part by the huge burden of diabetes and coronary artery disease. Young patients with ischemic cardiomyopathy and functional mitral regurgitation are being encountered more and more frequently. The eight articles in this section attempt to cover the spectrum of disorders seen in practice and the options available currently to conserve the mitral valve.

Professor Taweesak from Heart Institute, Thailand, addresses the challenges and evolution of repair in rheumatic etiology. He explains that better understanding of the mitral complex and dynamics have improved outcomes. The goal is to restore normal systolic and diastolic function, and even though isolated mitral regurgitation (MR) offers good hope, mixed lesions and stenosis are still a challenge. He reports an experience of 534 patients repaired in a 13-year period with very good outcomes. The physiologic and dynamic approach has significantly decreased the limitations of repair.

Professor Patrick Perier and his colleagues, in the review of repair in degenerative etiology (a predominantly type II dysfunction), stress the need to "Respect than Resect". In this article, he takes us through the various techniques to address the valve at different levels, stressing the point that a sound understanding is key to success. He suggests that posterior leaflet prolapse is not always simple and emphasizes that "excess tissue means extra skill". The four critical parameters on intra-operative echocardiographic assessment post-repair need attention of the reader.

Dr. Hartzell Schaffand and Dr. Anita Nguyen in their manuscript explain the Mayo Clinic experience of over 6000 cases of mitral repair over a 35-year period. The article recapitulates the various techniques described and highlights the triangular resection technique practiced at the Mayo. The philosophy has been very standard over the years, with resection for posterior leaflet and artificial chords for the anterior leaflet prolapse, stabilized with a flexible band annuloplasty. The results have 
been stellar, with a risk of reoperation over a 15 -year period being less than $6.9 \%$ in the over 1200 patients followed.

Ischemic mitral regurgitation is a surrogate marker of poor outcome. There are very limited studies and the CTSNet study published did not demonstrate difference in survival by correcting mitral regurgitation except for symptomatic benefit. It also showed that repair is better than replacement. However, the evidence was limited in terms of the numbers on which these conclusions were drawn.

Dr. Steven Bolling and his group from Ann Arbor proposed the undersized annuloplasty as the correction for this problem couple of decades ago. Dr. Bolling and Dr. Alexander Brescia in their article here focus on the pathophysiology, current evidence on surgical and trans-catheter techniques, and future directions. They make a strong pitch for intervention. They conclude that ischemic mitral regurgitation is not only a marker of dysfunction but also a target for intervention and propose that aggressive repair would improve outcomes. Dr. Pomar and Dr. Yadava in the video article on the same subject stress the importance of the alignment of the subvalvar apparatus and opine that there is no one-stop solution. They suggest that timing of intervention is more important than the technique.

Hypertrophic obstructive cardiomyopathy (HOCM) is commonly associated with MR, mostly due to systolic anterior motion (SAM) and the question of addressing the mitral valve is a common one.

Dr. Hartzell Schaff and the group at the Mayo Clinic have among the largest experience in the world, with more than 3000 cases treated by septal myectomy. The Society of Thoracic Surgeons (STS) database shows that close to one third of patients undergoing septal myectomy have the mitral valve addressed as compared to the Mayo experience of $4.9 \%$. Dr. Joon Hong and Dr. Schaff in their article clearly spell out how to address the mitral valve in this situation. They show with their experience that a complete septal myectomy to abolish SAM should be sufficient, and that the mitral valve needs to be addressed only if there is an intrinsic mitral lesion. The article takes the reader through the steps of septal myectomy and concomitant procedures on the mitral valve and sub-valvular apparatus.

Minimally invasive surgery (MIS) has been in practice for last 2 decades and there has been increasing interest recently with improved vision and technology. The Leipzig Heart Centre has been the pioneer in surgical and catheter innovations.

Dr. Piroze Davierwala and Dr. Mateo Cuartas from Leipzig review the status of minimal access mitral surgery today. The steep learning curve is a reality and there is a need for up to date evidence on MIS to recommend it. They suggest that patient selection, pre-operative screening, operative setup, and cannulation strategy are key to success. The article recapitulates various existing techniques and in addition mentions the Leipzig loop technique, concluding that the future is in reducing complexity and increasing reproducibility for better adaptation. Dr. Thilo Noack from the same group has provided a succinct review of all the trans-catheter techniques available today. He classifies them into four categories as coaptation devices, annuloplasty devices, chordal replacement systems, and left ventricular (LV) remodeling devices, and prophesies that the future is for the catheter therapy to move up from a palliative option to a definitive solution.

\section{Aortic valve}

Reparative techniques on the aortic valve are increasingly being used. While the mitral valve has a standardized method of classification and analysis, the aortic valve does not, posing difficulty to the surgeon. A better understanding of the dynamics and improved imaging have paved way for increasing adaptation of restorative techniques to the aortic valve.

Professor Joachim Schafers, in his unique article on the Geometry of Cusp and Root, describes the anatomy, nomenclature, and morphology of the annulus and sino tubular junction (STJ), and the most important measures - the effective height and geometric height (EH and GH). These two measurements have resulted in a paradigm shift in determining feasibility and adequacy of repair. He further states that the aortic valve should be considered a functional unit of cusp and root. Some of these concepts, including the role of annuloplasty, and the good and bad substrates for reparative surgery of the aortic valve are succinctly brought out in a video article by Schafers and Yadava.

Isolated valve repair was seldom attempted in the past, but two operations were defined to address root pathology in early 1990s which laid the foundation for modern reparative aortic work-The David operation in 1992 and the Yacoub operation in 1993.

The Brussels group under Professor Gebraine El Khoury is a strong proponent of the David operation also defined as the reimplantation technique. Dr. Laurent De Kerchove of the Brussels group shares in his original article their experience of 303 patients who underwent this procedure in tricuspid valve with an operative mortality, excluding acute dissections, of $0.3 \%$, over a 20 -year period. Freedom from reoperation was $90 \%$ at 10 years.

The other operation, the Yacoub, is otherwise called the remodeling technique. Dr. Schafers from Homburg presents his experience of 23 years with 1038 patients. He reports a similar freedom from reoperation of $92 \%$ at 10 years. He showed that bicuspids fared poorly and so did patients with annular dilatation. Currently, the authors recommend an annuloplasty if the annulus is greater than $25 \mathrm{~mm}$.

Professor Emmanuel Lansac from Paris in his article emphasizes the importance of annuloplasty and durability of repair. He discusses the different phenotypes and appropriate 
surgeries to recommend. He points out the importance of restoring the annulus to STJ ratio even in isolated valve repair if the annulus is $>25 \mathrm{~mm}$ and STJ $>30 \mathrm{~mm}$. The beautifully illustrated article describes the two-ring technique proposed by him - the annular and supra-annular.

The durability of these valve sparing procedures and the time required for the surgery versus a Bio-Bentall have been brought into question, especially in the older population.

Dr. Michael Borger and his colleagues from Leipzig Heart Centre in their interesting article looked at patients undergoing the David operation versus a bio Bentall over a 5-year period. They recommend that valve-sparing procedure should be attempted in anyone with suitable cusps. This study is retrospective and has limitations but initiates a very important argument.

Endocarditis was a definitive contraindication to valve repair techniques earlier, but with good results seen in the mitral valve, there is a limited scope in aortic conservation too.

Dr. Laurent Kerchove from the Brussels group again presents their experience with 42 patients over a 19-year period. They recommend considering repair if the lesions are limited or in tricuspid morphology. The Ozaki operation is a boon for valves that cannot be repaired. It is a replacement at the same time a reconstructive procedure-neocuspidization, with the major advantage being applicability to all ages and all types of pathology. Its role in congenital surgery has been very notable.

Dr. Christopher Baird from Boston Children's has provided a very special article focusing on the Ozaki procedure in children and its scope in association with small annulus and associated lesions as well. He opted to write specifically on this when we requested to share his experience of valve conservation in children. The article is beautifully illustrated and a very valuable addition to this issue.

\section{Tricuspid valve}

Tricuspid valve is commonly referred to as the "Forgotten valve". Like functional MR, tricuspid regurgitation (TR) is mostly functional and is a surrogate marker for poor outcome. The valve is also much more complex and problems may be congenital or acquired. Dr. Joe Dearani and Dr. Nishant Saran from the Mayo Clinic elucidate strategies for tricuspid repair and define the various techniques in their article. The underlying principle is being to look at the valve just like the mitral and address each component, not just the annulus. Dr. Joe Dearani who has a rich experience in cone repair for Ebstein's malformation also highlights this procedure in this review.

What is more common is the functional TR associated with left-sided disease. Dr. Aaron and Dr. Steven Bolling from University of Michigan outline the current thinking and the indications to address this very common and perplexing issue which appears simple, making a strong pitch for rigid and semi-rigid rings.

\section{Intra-operative trans-esophageal echocardiography}

We are all aware of the importance of trans-esophageal echocardiography (TEE), without which no repair procedure would be possible. Dr. Chandrasekaran Krishnaswamy from Mayo Clinic and Dr. Aayush Poddar have written an excellent review on the role of intraoperative TEE in simple terms. They have taken each condition individually and given the prebypass and post-bypass criteria for ease of understanding and to allow implementation in the operating room.

\section{Conclusion}

Reconstructive or reparative surgery should be the first choice in suitable valve morphology. Valve repair surgery had, in the past, only been the forte of a few exceptionally gifted surgeons. While the evolution of repair techniques has not been as rapid as one would expect, the last decade has seen a tremendous change, aided by advances in intraoperative echocardiography and superior anesthesiologic support.

Though valve replacement continues to be the most commonly performed procedure in our country, due to multifactorial reasons, growing evidence suggests that the mindset should change, and the option of repair should be given due consideration in each case. The surgeon must be able to justify to himself that repair was not feasible prior to discarding the option. An unsuccessful repair need not be a failure as one may always fall back on replacing the valve.

This special issue was conceived to provide readers with an up to date knowledge of the status of reparative surgery for common valvular problems so that they may reinvent themselves in this area, especially given the context of declining rheumatic burden and increasing degenerative and functional etiology. I am sure the content of this special issue, contributed by very select and highly experienced authors, will be highly valuable to your practice and knowledge. I sincerely thank the Editor in Chief for giving this opportunity and all the authors for providing the manuscripts written exclusively for this issue.

I hope you enjoy the good read.

"To raise new questions, new possibilities, to regard old problems from a new angle, requires creative imagination and marks real advance in science." - Albert Einstein 